0038-1098(95)00358-4

\title{
INFLUENCE OF OXYGEN CONTENT ON THE CATHODOLUMINESCENCE OF $\mathrm{YBa}_{2} \mathrm{Cu}_{3} \mathrm{O}_{7-x}$
}

\author{
P. Gómez* and J. Piqueras
}

Departamento de Física de Materiales, Facultad de Físicas, Universidad Complutense, 28040 Madrid, Spain and

M.J. Sayagués and J.M. González-Calbet

Departamento de Química Inorgánica, Facultad de Químicas, Universidad Complutense, 28040 Madrid, Spain

(Received 9 February 1995; in revised form 5 April 1995 by P. Burlet)

The influence of oxygen content of $\mathrm{YBa}_{2} \mathrm{Cu}_{3} \mathrm{O}_{7-x}$ ceramic samples on the cathodoluminescence emission has been studied in the scanning electron microscope. No luminescent band has been related to the presence of native oxygen vacancies in untreated samples. On the other hand vacancies are involved in luminescent centers formed after some treatments as electron irradiation.

Keywords: A. superconductors, C. scanning electron microscopy, D. optical properties, E. luminescence.

\section{INTRODUCTION}

LUMINESCENCE from $\mathrm{YBa}_{2} \mathrm{Cu}_{3} \mathrm{O}_{7-x}$ (YBCO) ceramics and crystals has been investigated by a number of authors in the past years. Some of the spectral features of YBCO luminescence were found to correspond with those observed in other high temperature superconductors (HTSC) such as $\mathrm{Bi}_{2} \mathrm{Sr}_{2} \mathrm{CaCu}_{2} \mathrm{O}_{x}$ (BSCCO) and $\mathrm{Tl}_{2} \mathrm{Ba}_{2} \mathrm{Cu}_{1} \mathrm{O}_{6+\delta}$ suggesting that luminescence bands are related to structural properties common to the mentioned materials, e.g. the existence of $\mathrm{Cu}-\mathrm{O}$ planes. Stankevitch et al. [1] reported experimental confirmation that YBCO and $\mathrm{BSCCO}$ show intrinsic luminescence at $2.85 \mathrm{eV}$ $(435 \mathrm{~nm})$ and attributed the emission to an oxygen quasimolecule formed in a $\mathrm{Cu}-\mathrm{O}$ plane. Several studies on cathodoluminescence $(\mathrm{CL})$ from $\mathrm{YBCO}$ have also shown the existence of emission bands in the blue-green spectral region [2-5]. In addition, CL observations in the scanning electron microscope indicate that oxygen depleted zones of YBCO emit a higher luminescence signal than oxygen rich regions [5-7]. In particular, it has been observed [8-10] that

* Also at Departamento de Física Aplicada, E.U.I.T.A., Universidad de Castilla-La Mancha, 13071 Ciudad Real, Spain. treatments causing oxygen deficiency in YBCO, such as vacuum annealing or electron irradiation, cause the appearance or the enhancement of a luminescence band at $2.3 \mathrm{eV}(530-540 \mathrm{~nm})$. The results indicated that this band is related to oxygen loss rather than to the presence of phases formed during the treatments. A luminescence band centered at the same energy has been observed by CL-microscopy in $\mathrm{Tl}_{2} \mathrm{Ba}_{2} \mathrm{Cu}_{1} \mathrm{O}_{6+\delta}$ and was related to local variations of oxygen content in the sample [11]. In BSCCO, electron irradiation in the scanning electron microscope produces the enhancement of several luminescence bands including the $2.3 \mathrm{eV}$ band [12]. The intrinsic luminescence of YBCO described in [1] has been also found to increase with electron irradiation in the scanning electron microscope [9]. The mentioned results on different HTSC indicate that changes in oxygen content and/ or distribution influence the material luminescence in the blue-green spectral region but the specific defects associated with some of the bands are not identified. In particular, thermal or irradiation treatments causing oxygen loss in YBCO can also involve electronic mechanisms generating complex defects with oxygen vacancies, with a certain analogy to the F-center formation in ionic crystals. In the present work the influence of oxygen content of YBCO on its luminescence properties is investigated by CL-microscopy. A 
series of samples prepared with different oxygen content were studied, both before any treatment and after controlled irradiation in a scanning electron microscope.

\section{EXPERIMENTAL METHOD}

The samples used were cut from sintered $\mathrm{YBa}_{2} \mathrm{Cu}_{3} \mathrm{O}_{7-x}$ disks with nominal $T_{c}$ value of $93 \mathrm{~K}$. Samples with different $x$ values were obtained by using a thermogravimetric analysis system (TGA) built on the basis of a Cahn D-200 electrobalance which operates under static atmosphere. A quartz hangdown tube and an electric furnace permit to operate from room temperature up to $1373 \mathrm{~K}$. A vacuum and a gas blending system were coupled to the TGA in order to work with a gas mixture at a given total pressure. $\mathrm{H}_{2} / \mathrm{He}$ mixtures were used as reducing atmospheres due to its low density. Due to the high sensitivity of the Cahn D-200 electrobalance, $x$ can be determined, for instance, within $\pm 2 \times 10^{-3}$ for a YBCO disk of about $100 \mathrm{mg}$. Temperature reduced program studies performed at a constant heating rate allow one to determine the oxygen content of the starting material as well as the existence of intermediate compounds from a plateau or inflection point of the so-obtained thermogram.

The absolute oxygen content was determined by reducing the samples under $\mathrm{H}_{2} / \mathrm{He}$ at $500^{\circ} \mathrm{C}$, a value of $x=0.1$ being obtained.

YBCO samples with $x=0.19,0.6,0.7,0.8$ and 0.9 were prepared under 100 mbar $\mathrm{H}_{2} / 400 \mathrm{mbar} \mathrm{He}$ heating up to the temperature needed to complete the reduction process from starting $\mathrm{YBa}_{2} \mathrm{Cu}_{3} \mathrm{O}_{7-x}$ to the $x$ value required. Then the sample is annealed at constant temperature under $500 \mathrm{mbar} \mathrm{He}$. Finally, the sample is cooled to room temperature inside the balance.

The samples were observed in a Hitachi S-2500 scanning electron microscope. The emissive and CL mode were used at accelerating voltages of $20 \mathrm{keV}$ and at temperatures ranging from 80 to $300 \mathrm{~K}$. The light arising from the sample (CL signal) was concentrated by an optical lens on a light guide which was attached either to a photomultiplier, in order to get the CL image, or to a monochromator (Oriel 78215), in order to get the spectral distribution of the $\mathrm{CL}$ signal. Sample temperature was controlled and recorded using an Oxford Instruments ITC-4 temperature controller.

The samples were placed in the same specimen holder in order to perform the CL measurements under the same experimental conditions.

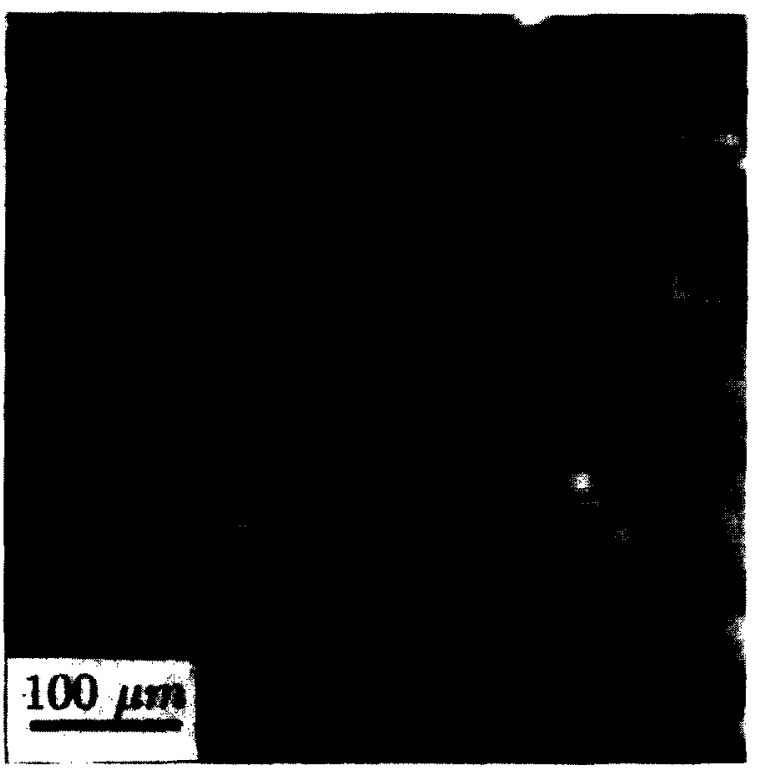

Fig. 1. CL distribution on the 0.19 sample. Emission is inhomogeneous in the sample but the appearance of periodicity is an electronic artifact.

\section{RESULTS AND DISCUSSION}

Results on CL intensity and spectral features of observed luminescence were similar in all the investigated untreated samples, independent of oxygen content. CL intensity shows an inhomogeneous distribution with dark and bright regions (Fig. 1). The periodicity observed in Fig. 1 is an electronic artifact appearing because of the low signal level in untreated samples. Spectra recorded in different regions of the sample reveal the existence of several bands with peaks in the range $400-600 \mathrm{~nm}$. Figure 2

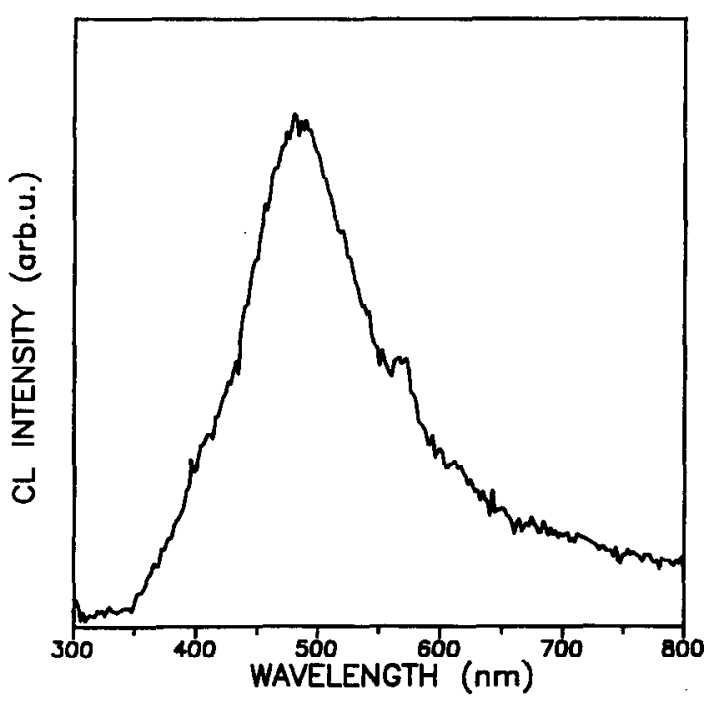

Fig. 2. CL spectra from a bright area of the 0.19 sample. 


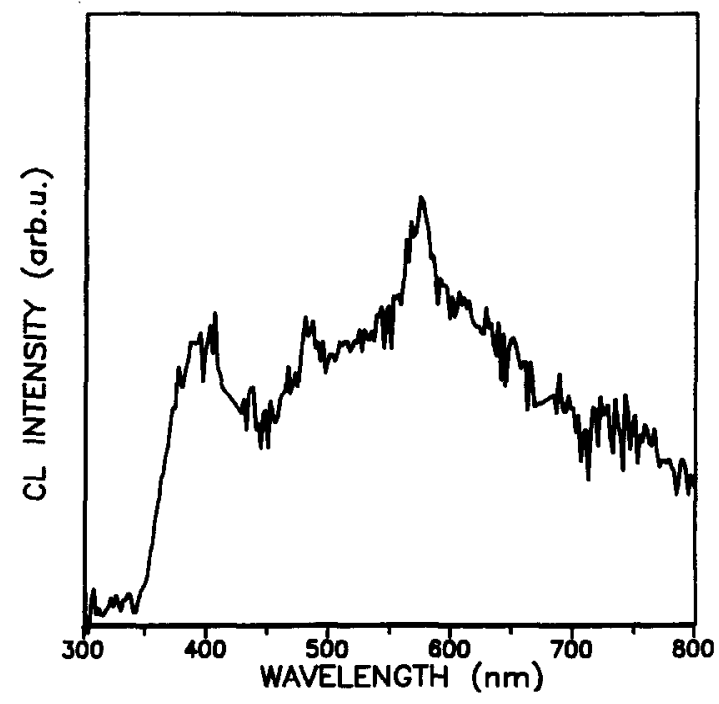

Fig. 3. CL spectra from a dark area of the 0.19 sample.

shows the spectrum in a bright region with bands centered at about $400 \mathrm{~nm}, 470-480 \mathrm{~nm}$ and $570 \mathrm{~nm}$. In this case one of the luminescence bands is maximized. Figure 3 shows a spectrum recorded in one of the darker regions of the sample. The emission has a complex spectral structure with a resolved band at about $400 \mathrm{~nm}$ and a very broad band peaked at about $550 \mathrm{~nm}$.

As mentioned above it has been found in previous works that oxygen depleted regions show a higher luminescence signal than oxygen rich regions and that treatments causing oxygen deficiency also cause the enhancement of the $2.3 \mathrm{eV}(530 \mathrm{~nm})$ band. The present results do not show differences in the observed luminescence as a function of oxygen content. This discrepancy can be explained if the oxygen related luminescence band does not depend only on the oxygen content, but on the treatments - annealing or electron irradiation - able to modify the oxygen content and to form some complex defects involving, for instance, oxygen vacancies. In order to investigate this possibility all samples were irradiated under the same conditions in the scanning electron microscope and the total luminescence intensity after irradiation was recorded. The luminescence intensity increased in all samples but no relationship between oxygen content in the sample and final luminescence intensity was observed. The quantitative comparison appeared to be difficult and not reliable, partly because the original $\mathrm{CL}$ intensity is inhomogeneous in the sample and the quantitative evolution with time is a function of the region considered. For this reason the influence of oxygen content on luminescence was studied by monitoring the spectral changes taking place during electron irradiation. The result obtained is repre-

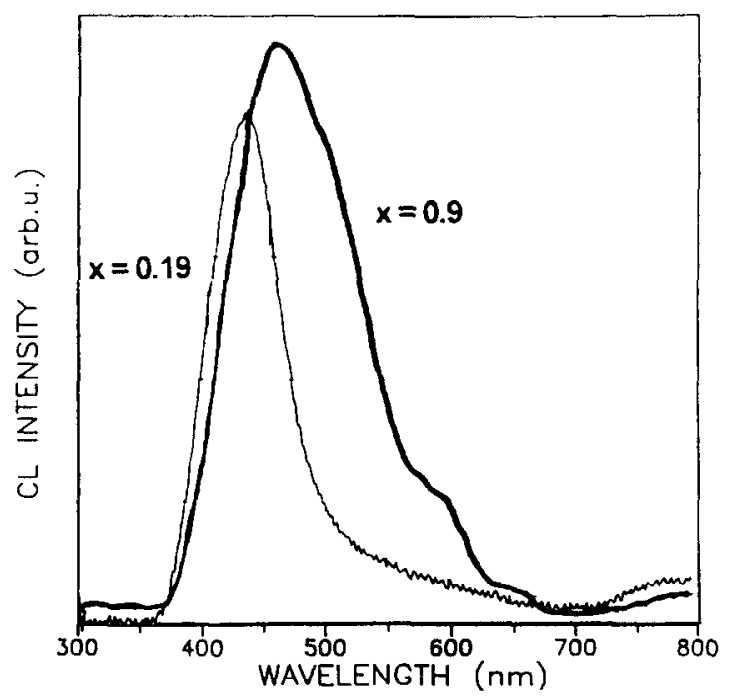

Fig. 4. CL spectra from irradiated samples.

sented in Fig. 4. In these spectra the inhomogeneity has been averaged by recording the emissions from an area including bright and dark regions. On the other hand the high luminescence increase during irradiation tends to reduce the relative emission differences among the regions. The spectra shown in Fig. 4 are a consequence of the complex character of the original emission, shown in Figs 2 and 3, and of the different behaviour of the samples during the irradiation. The sample with the higher oxygen content $(x=0.19)$ shows only a strong luminescence band centered at about $420 \mathrm{~nm}(2.95 \mathrm{eV})$ while the other samples $(x=0.6-0.9)$ show a shoulder, in the range 490$530 \mathrm{~nm}(2.5-2.3 \mathrm{eV})$ and a band at about $600 \mathrm{~nm}$ $(2.06 \mathrm{eV})$. A correlation between the value of $x$ and the relative intensity of the $490-530 \mathrm{~nm}$ emission was not observed. This result shows that oxygen deficiency favours the growth of the luminescence at about $500 \mathrm{~nm}$ during irradiation. Comparison with spectra of unirradiated samples indicates that the intensity increase of the luminescence bands during irradiation is sample dependent. In the sample with high oxygen content the $400-420 \mathrm{~nm}$ band drastically increases and becomes the only resolved band in the spectrum. This band corresponds to the blue intrinsic luminescence reported in previous PL $[1,8]$ and $C L$ [9] works. In samples with lower oxygen content there is also a marked increase of luminescence in the range $450-550 \mathrm{~nm}$ causing the appearance of a shoulder or a resolved band at $500-530 \mathrm{~nm}$. This emission was previously detected [8-10] in YBCO samples which had received treatments causing oxygen loss as well as in samples of $\mathrm{BSCCO}$ [12] and $\mathrm{Tl}_{2} \mathrm{Ba}_{2} \mathrm{Cu}_{1} \mathrm{O}_{6+\delta}$ [11].

It appears that during irradiation, different luminescence bands increase and consequently the band at 


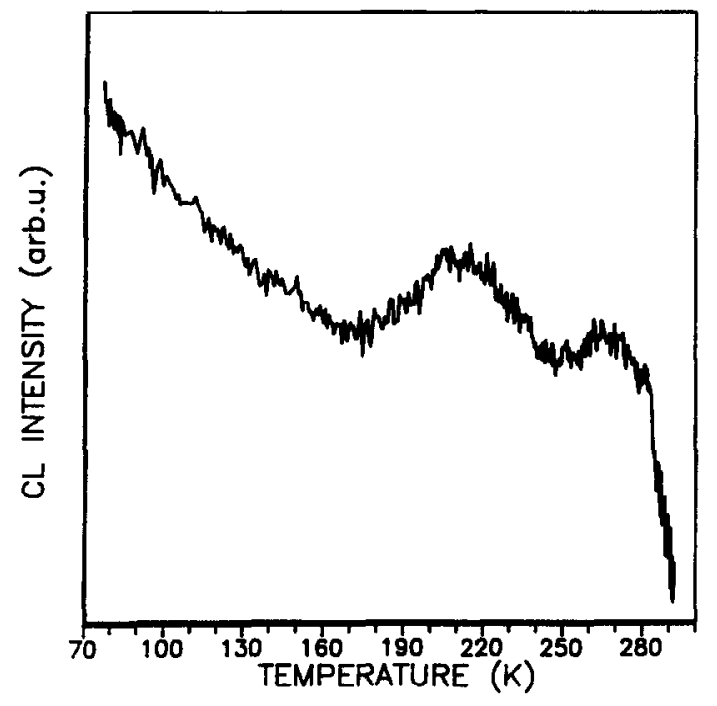

Fig. 5. Variation of luminescence intensity with temperature when cooling down the sample.

$530 \mathrm{~nm}$ observed in the oxygen deficient samples can have a complex character. Recent time resolved photoluminescence measurements [10] in which two decay times have been observed in the $530 \mathrm{~nm}$ band, support this possibility.

Temperature dependence of CL and PL intensity of YBCO has been previously reported $[3,4,7,10]$. The dependence can show a complex behaviour due to the different bands involved. Yaskolko et al. [4] concluded that $\mathrm{CL}$ is associated with a phase transition of order-disorder type in the oxygen sublattice and Luff et al. [3] detected intensity changes at temperatures at which anomalies of other physical properties were observed. In order to study the possible relationship between temperature dependence of $\mathrm{CL}$ and mechanisms occuring in the oxygen sublattice the temperature dependence of the panchromatic CL was measured in all samples used in this work. Besides the general trend of luminescence increase by decreasing temperature some steps were observed in the temperature dependence curve. In the oxygen deficient samples the curves were in general not reproducible. On the contrary, the high oxygen content sample showed in different runs the dependence shown in Fig. 5. In particular the bumps at about $220 \mathrm{~K}$ and $270 \mathrm{~K}$ were reproducible. Further work and comparison with the temperature dependence of other physical properties are necessary to determine if these features are related to fine structural changes in the sample.

\section{CONCLUSIONS}

$\mathrm{CL}$ emission of untreated YBCO has a complex character with a resolved emission band at about 400 $420 \mathrm{~nm}$ and a complex emission in the spectral range 450-600 nm. Spectral features appear to be independent of oxygen content. During irradiation with the microscope electron beam the evolution of the spectrum depends on the oxygen content of the sample. In oxygen deficient samples spectra show a shoulder or a resolved band at about $550-530 \mathrm{~nm}$ while in samples with high oxygen content only the $420 \mathrm{~nm}$ band is observed. Centers causing the $530 \mathrm{~nm}$ emission involve oxygen vacancies but are formed only after irradiation or annealing treatments. Native vacancies in the untreated samples are not related to this luminescence band.

Acknowledgement - This work has been partly supported by DGICYT (Project PB93-1256).

\section{REFERENCES}

1. V.G. Stankevitch, N.Yu Svechnikov, K.V. Kaznacheev, M. Kamada, S. Tanaka, S. Hirose, R. Kink, G.A. Emel'chenko, S.G. Karabachev, T. Wolf, H. Berger \& F. Levy, Phys. Rev. B42, 1024 (1993).

2. V.N. Andreev, B.P. Zakharchenya, S.E. Nikitin, F.A. Chudnovskii, E.B. Shadim \& E.M. Ster, JETP Lett. 46, 492 (1987).

3. B.J. Luff, P.D. Townsend \& J. Osborne, J. Phys. D. Appl. Phys. 21, 663 (1988).

4. V.Ya Yaskolko, L.N. Oster \& K.M. Mukinov, Phys. Status Solidi (a) 123, K35 (1991).

5. J. Piqueras, P. Fernández \& J.L. Vicent, Appl. Phys. Lett. 57, 2722 (1990).

6. J.H. Miller Jr., J.D. Hunn, S.L. Holder \& A.N. DiBianca, Appl. Phys. Lett. 56, 89 (1990).

7. F. Domínguez-Adame, P. Fernández, J. Piqueras, P. Prieto, C. Barrero and M.E. Gómez, J. Appl. Phys. 71, 2778 (1992).

8. A. Remón, J.A. García, P. Gómez, J. Piqueras and F. Dominguez-Adame, Phys. Stat. Sol. (a) 136, K127 (1993).

9. P. Gómez, J. Jímenez, P. Martín, J. Piqueras and F. Domínguez-Adame, J. Appl. Phys. 74, 6289 (1993).

10. J.A. García, A. Remón and J. Piqueras, Phys. Stat. Sol. (a), 144, 217 (1994).

11. P. Gómez, J. Piqueras and C. Opagiste, Solid State Commun. 91, 747 (1994).

12. A. Torres, J. Jiménez, P. Gómez and J. Piqueras, Materials Research Society, Fall Meeting, Boston, November 1994. 